\title{
Short-term load forecasting of the distribution system using cuckoo search algorithm
}

\author{
Saroj Kumar Panda ${ }^{1}$, Papia Ray ${ }^{1}$, Debani Prasad Mishra ${ }^{2}$, Surender Reddy Salkuti ${ }^{3}$ \\ ${ }^{1}$ Department of Electrical Engineering, Veer Surendra Sai University of Technology (VSSUT), Odisha, India \\ ${ }^{2}$ Department of Electrical Engineering, International Institute of Information Technology (IIIT), Bhubaneswar, India \\ ${ }^{3}$ Department of Railroad and Electrical Engineering, Woosong University, Daejeon, Republic of Korea
}

\begin{tabular}{l} 
Article Info \\
\hline Article history: \\
Received Jan 19, 2021 \\
Revised Jan 19, 2022 \\
Accepted Jan 24, 2022 \\
\hline Keywords: \\
Artificial neural network \\
Back propagation \\
Cuckoo search \\
Distribution system \\
Optimization \\
Short term load forecasting
\end{tabular}

\begin{abstract}
For solving the different optimization problems, the cuckoo search is one of the best nature's inspired algorithms. It is an effective technique compare to other optimization methods. For this manuscript, we are using a back propagation neural network for the Xintai power plant consist of short-term electrical load forecasting. The limitation of back propagation is overcome by the cuckoo search algorithm. The function is used for cuckoo search is Gamma probability distribution and its result is compared with other possible cuckoo search methods. The mean average percentage error of Gamma cuckoo search is $0.123 \%$, cuckoo search with Pareto based is $0.127 \%$ and Levy based cuckoo search is $0.407 \%$. Other results of the cuckoo search are also found by a linear decreasing switching parameter with a mean average error is $0.344 \%$ and $0.389 \%$ of mean average error with the use of an exponentially increasing switching parameter. This improved cuckoo search algorithm brings good results in the predicted load which is very important for the Xintai power plant using short-term load forecasting.
\end{abstract}

This is an open access article under the CC BY-SA license.

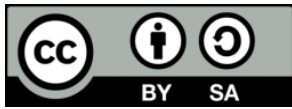

\section{Corresponding Author:}

Surender Reddy Salkuti

Department of Railroad and Electrical Engineering, Woosong University

17-2, Jayang-Dong, Dong-Gu, Daejeon - 34606, Republic of Korea

Email: surender@wsu.ac.kr

\section{INTRODUCTION}

For a given problem, the optimal solution is obtained by a systematic procedure which is known as optimization [1]-[3]. It is used for the solution of maximum and minimum value of a problem and it is called cost function or objective function. There are two types of optimization problems, i.e., constrained and unconstrained problems. For the solution of all subsets, constrained problems are using and for all viable solutions, unconstrained functions are using [4]-[6]. Now a day, the optimization technique is adopted by different areas but not limited to specific systems. Like the transmission of electricity with a minimum loss, design of the system, operation of an electric circuit, generation of electricity and wireless communication routing. So, suitable optimization is required for the calculation of the computation time, converge rate and minimum or maximum value accurately [7], [8].

Nature's inspired algorithm is constructed by the researcher with the inspection of the behavior of animals. For the calculation of the distance between a bat and its surrounding, the researchers are using a bat-inspired algorithm [9]. This technique is also used for the calculation object in frequency tuning. Similarly, another nature's inspired algorithm is particle swarm optimization (PSO) where the fishes and birds are searching for their food considered as a potential solution in PSO [10]. In this technique, the animals are searching for food and they communicate the food to the rest of the group when the food source is found. Here, the food source is considered as the best solution for the processing of food among groups. 
For the calculation of storm and prince, the differential evolution (DE) algorithm is using based on a population vector. This population vector consists of the size of the population which does not change during the searching process and uniform probability distribution. The different parameters are affecting the growth of the population i.e. mutation (new generation), crossover (increasing of diversity) and selection (finding of new solution). It is a robust and efficient process used for continuous space [11]. The behavior of foraging is used by Ant and Bee algorithm which is known as a chemical messenger. It is also known as pheromone [12]. For global optimization, the use of nature's inspired algorithm is simulated annealing (SA). This technique finds a good solution as compared to the limited time constraint of the global solution [13].

The other nature's inspired algorithm is the cuckoo search (CS) algorithm which depends on the reproduction of the kids to increase the population [14]. But, this algorithm is good as compare to other algorithms because the other algorithms like DE, SA and PSO are derived from the CS algorithm has potential random walk and makes the balance between local and global search as compared to SA and GA [14]. The CS algorithm is better than the DE algorithm in terms of convergence speed and finding a good solution [15]. The computational efficiency of the CS algorithm is also good as compared to the PSO algorithm. The CS algorithm is also used in the smart grid for the minimization of loss of real power by control of fault and variation of voltage with allowable level [16]. So, with the consideration of time from one hour to one week, short term load forecasting (STLF) is using in industries. It is used for the planning and maintenance of power networks [17]. The factors which affect the STLF are considered for its work in [18].

The research gap from the above study is the old techniques are bringing poor results in STLF in past. So, in this manuscript, the research gap is fulfilled by the application of different distribution of cuckoo search algorithms in STLF which removes the disadvantages of old techniques. The other parts of this manuscript are arranged as follows: section 2 gives the simulation of STLF. Section 3 presents results and discussions of the work. At last, the conclusion of the work is represented by section 4 .

\section{STLF SIMULATION}

It brings the results of the forecasted load in STLF. After that, the forecasted load will compare with the actual load. Then, we applied the mean absolute percentage error (MAPE) to calculate the error in the forecasted load as given in (1).

$$
M A P E=\frac{1}{N} \sum_{i=1}^{N}\left(\frac{\text { Actual load }- \text { forecasted load }}{\text { Actual load }}\right) \times 100
$$

Where $N$ is total number of the data set.

\subsection{Collection of data}

The historical data is collected from the Xintai power plant in the year 2016 from the date of 6.10 to 6.30. The data set is divided into three parts i.e. training, validation and testing as in [19]. Here sunny day is expressed by 0 , cloudy day by 0.5 and rainy day by 1 .

\subsection{Pre-processing of data}

The transfer function depends on the input value. If the input value is very large, then the output value does not contain the actual value. So it is avoided by set-up the normalized value within the range of $[0,1]$ using the minimap function in MATLAB 2015 software package. The processing data is also considered for the missing data.

\subsection{Simulation result}

The STLF using a feed-forward neural network (FFNN) [20]-[23] is shown in Figure 1. It contains 4 inputs, 25 hidden layers and one output layer with a transfer function. Here, the sigmoid function is used as a transfer function. Figure 1 explains the process of the data transformation from the input layer to the output layer through hidden layers. It also helps in the prediction of the forecasted load. The hourly based load is forecasted at the output of NN [24] uses Levenberg-Marquardt [25] back propagation for the training and forecasts.

Figure 2 shows the flow chart of a hybrid Levenberg-Marquardt. It is good as compared to LevenbergMarquardt BP. Because BP [26]-[29] able to finds the minimum but it will not able to find the global minima in test function or loss. Figure 2 explains that, the feasibility solution of the forecasted load [30]-[32]. It helps in the removal of the disturbance signal present in the data set for the smooth train of FFNN. 


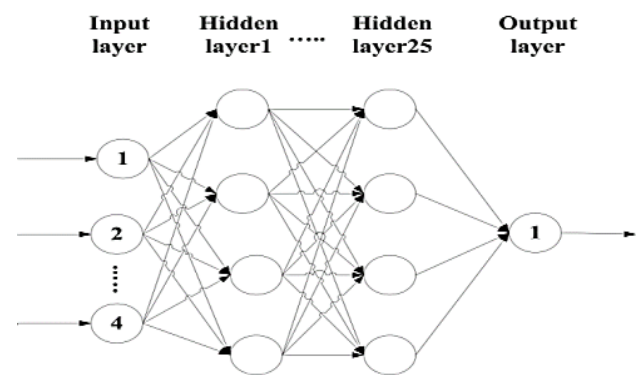

Figure 1. FFNN with BP

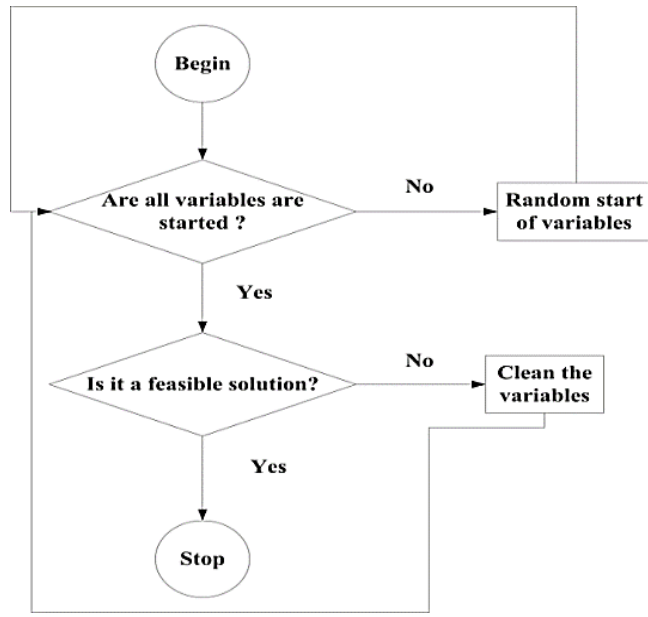

Figure 2. Flow chart of hybrid Levenberg-Marquardt

\section{RESULTS AND DISCUSSION}

The load forecasting is considered from 6.10 to 6.30 in the year 2016 which contains probability CS is given in Tables 1 and 2 and the Cauchy switched parameter CS algorithm is given in Tables 3 and 4 . Table 5 represents a comparison of different techniques in STLF [33], [34]. Figures 3 to 8 present the analysis of actual and forecasted load.

Table 1. Load forecasting with probability CS algorithm

\begin{tabular}{cccccc}
\hline $\begin{array}{c}\text { Actual load } \\
(\text { MW) }\end{array}$ & LevyCS & CauchyCS & GaussCS & GammaCS & ParetoCS \\
\hline 943 & 945 & 942 & 941 & 943 & 942 \\
914 & 915 & 913 & 912 & 914 & 913 \\
907 & 903 & 904 & 905 & 907 & 906 \\
875 & 865 & 875 & 875 & 870 & 874 \\
873 & 860 & 869 & 870 & 870 & 871 \\
872 & 871 & 870 & 869 & 872 & 871 \\
931 & 929 & 930 & 929 & 930 & 930 \\
976 & 972 & 976 & 973 & 975 & 975 \\
1062 & 1061 & 1061 & 1059 & 1062 & 1060 \\
1144 & 1140 & 1140 & 1141 & 1144 & 1142 \\
1213 & 1210 & 1209 & 1210 & 1213 & 1211 \\
1263 & 1258 & 1262 & 1260 & 1260 & 1262 \\
1231 & 1230 & 1230 & 1229 & 1230 & 1230 \\
1196 & 1185 & 1195 & 1193 & 1190 & 1195 \\
1150 & 1145 & 1148 & 1147 & 1150 & 1148 \\
1190 & 1185 & 1187 & 1185 & 1188 & 1188 \\
1212 & 1207 & 1210 & 1208 & 1210 & 1210 \\
1231 & 1226 & 1229 & 1225 & 1230 & 1230 \\
1223 & 1221 & 1221 & 1219 & 1223 & 1221 \\
1228 & 1223 & 1226 & 1223 & 1225 & 1226 \\
1245 & 1240 & 1242 & 1242 & 1240 & 1243 \\
1317 & 1315 & 1314 & 1313 & 1317 & 1315 \\
1214 & 1210 & 1211 & 1212 & 1214 & 1213 \\
1081 & 1075 & 1080 & 1079 & 1081 & 1080 \\
\hline
\end{tabular}


Table 2. MAPE with probability CS algorithm

\begin{tabular}{cc}
\hline CS algorithms & MAPE (\%) \\
\hline LevyCS & 0.407 \\
CauchyCS & 0.168 \\
GaussCS & 0.264 \\
GammaCS & 0.123 \\
ParetoCS & 0.127 \\
\hline
\end{tabular}

Table 1 explains the results of the different distribution of the cuckoo search. It also helps to know that, the proposed distribution is good for STLF. The predicted loads are very important for STLF which controls the price of electricity. Table 2 explains the MAPE results of the different distribution of cuckoo search. It also helps to know that, the proposed distribution is good for STLF which also gives less error in forecasted load. It indicates the load stability of different methods.

Table 3 explains the results of the different distribution of the cuckoo search. It also helps to know that, the proposed distribution is good for STLF. The predicted loads are very important for STLF which controls the price of electricity. Table 4 explains the MAPE results of the different distribution of cuckoo search. It also helps to know that, the proposed distribution is good for STLF which also gives less error in forecasted load. It indicates the load stability of different methods. Table 5 explains the MAPE results of different methods used for STLF and it brings high error in STLF as compare to different distribution functions of CS. So the CS is good for STLF which gives less error in forecasted load.

The MAPE of Gamma-CS is $0.123 \%$ as compared to the MAPE of Pareto-CS is $0.127 \%$ as given in Table 2. So the result of Pareto based CS is better than Levy probability CS. The performance of Levy CS is the least as compared to the other four probability methods. The result of decreasing the switching parameter in CS (CSLD) with respect to MAPE is $0.344 \%$ and the result of exponentially increasing parameter in CS (CSEI) with respect to MAPE is $0.389 \%$. So the increasing switching parameter brings good results as compared to constant real switching parameters of CS (CSCo) as given in Table 4 and Gamma-CS is also good as compared to other techniques as given in Table 5.

Table 3. Load forecasting with switching parameter CS algorithm

\begin{tabular}{cccccc}
\hline Actual load & \multicolumn{5}{c}{ Forecasted load (MW) } \\
(MW) & CSCo & CSLD & CSLI & CSPI & CSEI \\
\hline 943 & 909 & 911 & 910 & 911 & 912 \\
914 & 908 & 912 & 910 & 912 & 913 \\
907 & 895 & 905 & 900 & 905 & 906 \\
875 & 849 & 870 & 850 & 872 & 873 \\
873 & 855 & 870 & 860 & 870 & 871 \\
872 & 855 & 870 & 860 & 869 & 870 \\
931 & 915 & 930 & 920 & 929 & 930 \\
976 & 961 & 970 & 960 & 970 & 973 \\
1062 & 1051 & 1060 & 1050 & 1060 & 1061 \\
1144 & 1132 & 1140 & 1130 & 1142 & 1143 \\
1213 & 1211 & 1212 & 1210 & 1211 & 1212 \\
1263 & 1262 & 1262 & 1260 & 1259 & 1260 \\
1231 & 1226 & 1230 & 1225 & 1230 & 1231 \\
1196 & 1193 & 1195 & 1190 & 1192 & 1195 \\
1150 & 1141 & 1149 & 1140 & 1130 & 1149 \\
1190 & 1181 & 1189 & 1180 & 1183 & 1185 \\
1212 & 1206 & 1210 & 1205 & 1209 & 1210 \\
1231 & 1228 & 1230 & 1225 & 1228 & 1229 \\
1223 & 1216 & 1220 & 1215 & 1211 & 1213 \\
1228 & 1222 & 1227 & 1220 & 1225 & 1226 \\
1245 & 1231 & 1240 & 1230 & 1234 & 1235 \\
1317 & 1307 & 1310 & 1305 & 1308 & 1309 \\
1214 & 1206 & 1210 & 1205 & 1205 & 1208 \\
1081 & 1072 & 1080 & 1070 & 1074 & 1075 \\
\hline
\end{tabular}

Table 4. MAPE with switching parameter CS algorithm

\begin{tabular}{cc}
\hline CS algorithms & MAPE $(\%)$ \\
\hline CSCo & 0.895 \\
CSLD & 0.344 \\
CSLI & 0.957 \\
CSPI & 0.574 \\
CSEI & 0.389
\end{tabular}


Table 5. MAPE with different algorithms

\begin{tabular}{lc}
\hline \multicolumn{1}{c}{ Algorithms } & MAPE (\%) \\
\hline Back propagation neural network (BPNN) & 3.42 \\
Genetic algorithm back propagation neural network (GA-BPNN) & 3.86 \\
Particle swarm optimization Elman neural network (PSO-ENN) & 1.17 \\
\hline
\end{tabular}

Figure 3 (a) gives the forecasted load of the LevyCS distribution of cuckoo search. This method brings good accuracy and maintains load stability. It is a robust method which includes all variable affect the load in a short interval of time and gives less error in output with the use of MAPE calculation. Figure 3 (b) gives the forecasted load of the CauchyCS distribution of cuckoo search. This method brings good accuracy and maintains load stability. It is a robust method which includes all variable affect the load in a short interval of time and gives less error in output with the use of MAPE calculation.

Figure 4 (a) gives the forecasted load of the GaussCS distribution of cuckoo search. This method brings good accuracy and maintains load stability. It is a robust method which includes all variable affect the load in a short interval of time and gives less error in output with the use of MAPE calculation. Figure 4 (b) gives the forecasted load of the GammaCS distribution of cuckoo search. This method brings good accuracy and maintains load stability. It is a robust method which includes all variable affect the load in a short interval of time and gives less error in output with the use of MAPE calculation.

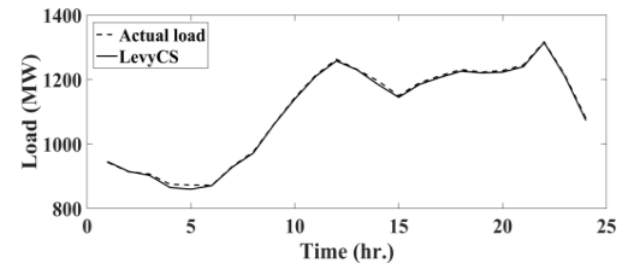

(a)

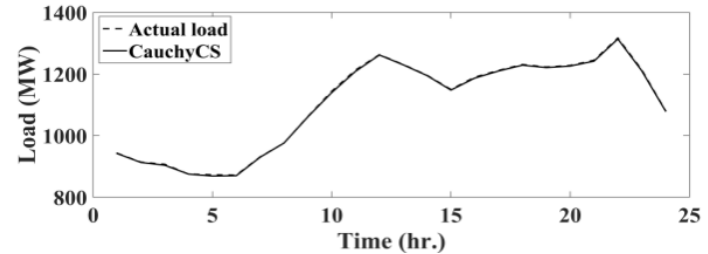

(b)

Figure 3. Comparison between actual and predicted loads (a) using LevyCS method and (b) using CauchyCS method

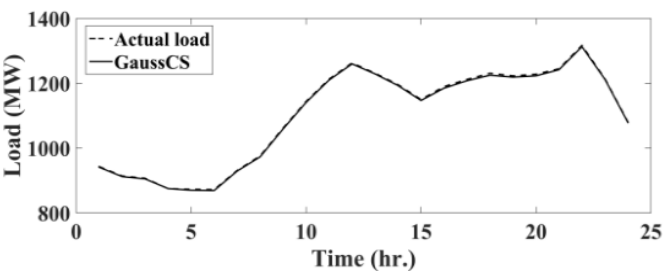

(a)

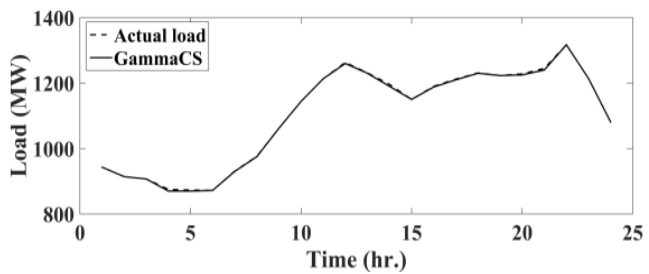

(b)

Figure 4. Comparison between actual and predicted loads (a) using GaussCS method and (b) using GammaCS method

Figure 5 (a) gives the forecasted load of the Pareto distribution of cuckoo search. This method brings good accuracy and maintains load stability. It is a robust method which includes all variable affect the load in a short interval of time and gives less error in output with the use of MAPE calculation. Figure 5 (b) gives the forecasted load of the different distribution of cuckoo search. These methods are bringing good accuracy and maintain load stability. These are robust methods which include all variable affect the load in a short interval of time and give less error in output with the use of MAPE calculation.

Figure 6 (a) gives the forecasted load of the CSCo distribution of cuckoo search. This method brings good accuracy and maintains load stability. It is a robust method which includes all variable affect the load in a short interval of time and gives less error in output with the use of MAPE calculation. Figure 6 (b) gives the forecasted load of the CSLD distribution of cuckoo search. This method brings good accuracy and maintains load stability. It is a robust method which includes all variable affect the load in a short interval of time and gives less error in output with the use of MAPE calculation. 


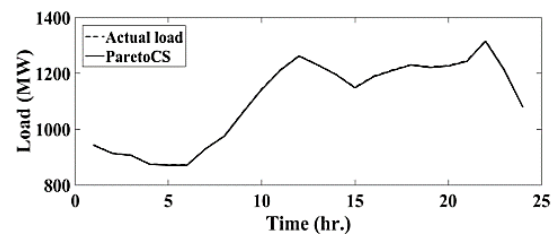

(a)

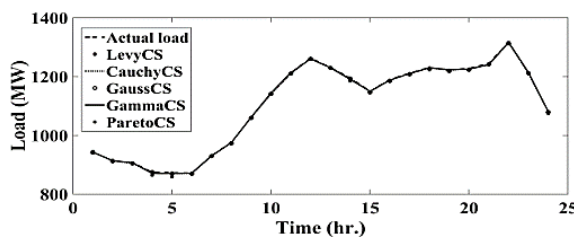

(b)

Figure 5. Comparison between actual and predicted loads: (a) using ParetoCS method and (b) using different methods

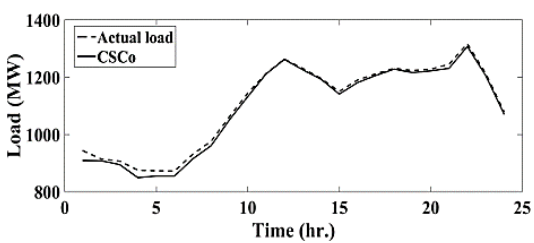

(a)

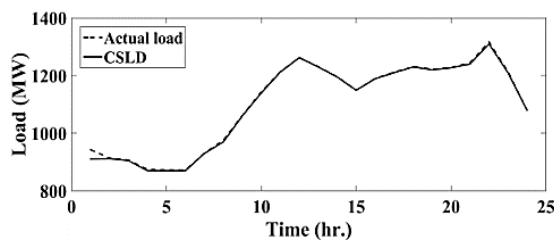

(b)

Figure 6. Comparison between actual and predicted loads: (a) using CSCo method and (b) using CSLD method

Figure 7 (a) gives the forecasted load of the CSLI distribution of cuckoo search. This method brings good accuracy and maintains load stability. It is a robust method which includes all variable affect the load in a short interval of time and gives less error in output with the use of MAPE calculation. Figure 7 (b) gives the forecasted load of the CSPI distribution of cuckoo search. This method brings good accuracy and maintains load stability. It is a robust method which includes all variable affect the load in a short interval of time and gives less error in output with the use of MAPE calculation. Figure 8 (a) gives the forecasted load of the CSEI distribution of cuckoo search. This method brings good accuracy and maintains load stability. It is a robust method which includes all variable affect the load in a short interval of time and gives less error in output with the use of MAPE calculation. Figure 8 (b) gives the forecasted load of the different distribution of cuckoo search. These methods are bringing good accuracy and maintain load stability. These are robust methods which include all variable affect the load in a short interval of time and give less error in output with the use of MAPE calculation.

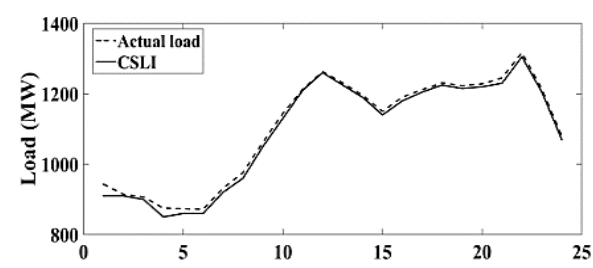

(a)

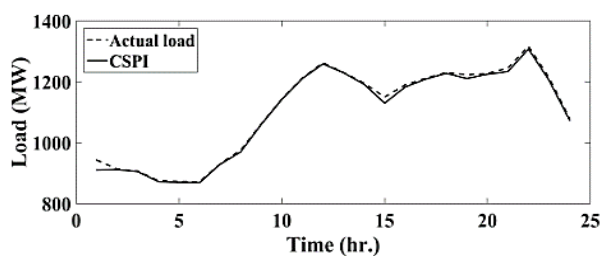

(b)

Figure 7. Comparison between actual and predicted loads (a) using CSLI method and (b) using CSPI method

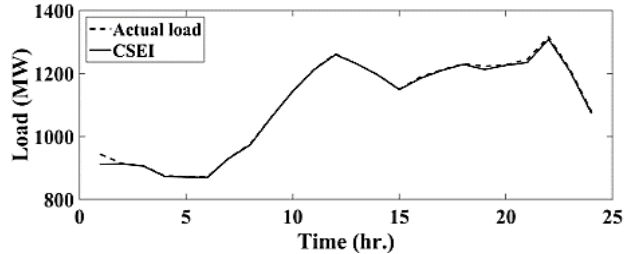

(a)

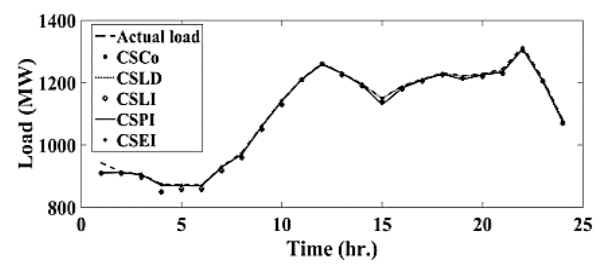

(b)

Figure 8. Comparison between actual and predicted loads (a) using CSEI method and (b) using different methods 


\section{CONCLUSION}

The optimization theory and its importance in the engineering problem are described in this manuscript. The different nature's inspired algorithms like PSO, DE and SA are discussed with their work. Here we got good results in efficient random work of the CS algorithm and maintained the balance between the local and global random walk as compared to other algorithms. It is also reviewed the work of NN for the STLF. The CS for improved BP is also discussed. The probability distribution and dynamic switching parameters are also discussed for the improvement of CS. For the electric load forecasting, 4-25-1 FFNN is used. The Gamma probability rings good results as compared to other methods and its error is $0.123 \%$. The average error of Pareto and Levy based CS is $0.127 \%$ and $0.407 \%$. The average error of decreasing switching parameter is $0.344 \%$ in CS and it is good as compared to exponentially increasing parameters i.e. $0.389 \%$.

\section{ACKNOWLEDGEMENTS}

This research work was funded by "Woosong University’s Academic Research Funding - 2022".

\section{REFERENCES}

[1] Y. Wang and C. Wei, "Design optimization of office building envelope based on quantum genetic algorithm for energy conservation," Journal of Building Engineering, vol. 35, no. 102048, pp. 1-15, 2021, doi: 10.1016/j.jobe.2020.102048.

[2] B. P. Sahoo and S. Panda, "Improved grey wolf optimization technique for fuzzy aided PID controller design for power system frequency control," Sustainable Energy, Grids and Networks, vol. 16, pp. 278-299, 2018, doi: 10.1016/j.segan.2018.09.006.

[3] S. Noureddine, "An Optimization Approach for the Satisfiability Problems," Applied Computing and Imformatics, vol. 11, no. 1, pp. 47-59, 2015, doi: 10.1016/j.aci.2011.11.002.

[4] R. D. Tordecilla, A. A. Juan, J. R. Montoya-Torres, C. L. Quintero-Araujo, and J. Panadero, "Simulation-optimization methods for designing and assessing resilient supply chain networks under uncertainty scenarios: A review," Simulation Modelling Practice and Theory, vol. 106, no. 102166, pp. 1-10, 2021, doi: 10.1016/j.simpat.2020.102166.

[5] P. Amaral, C. Duarte, and P. Costa, "On the impact of timer resolution in the efficiency optimization of synchronous buck converters,” Int. J. of Power Elect. and Drive Systems, vol. 6, no. 4, pp. 693-702, 2015, doi: 10.11591/ijpeds.v6.i4.pp693-702.

[6] G. W. Yen, A. Monadi, N. R. N. Idris, A. Jidin, and T. Sutikno, "Proposed voltage vector to optimize efficiency of direct torque control," Int. J. of Power Electr. and Drive Systems, vol. 4, no. 4, pp. 578-586. 2014, doi: 10.11591/ijpeds.v4i4.6918.

[7] R. G. Omar, "Coast function parameters optimization for DC battery sourcse inverter feeding three-phase inductive," Int. J. of Power Electr. and Drive Sys., vol. 11, no. 4, pp. 1799-1804, 2020, doi: 10.11591/ijpeds.v11.i4.pp1799-1804.

[8] Y. Babkrani, A. Naddami, and M. Hilal, "A smart cascaded H-bridge multilevel inverter with an optimized modulation techniques increasing the quality and reducing harmonics," International Journal of Power Electronics and Drive Systems, vol. 10, no. 4, pp. 1852-1862, 2019, doi: 10.11591/ijpeds.v10.i4.pp1852-1862.

[9] X. S. Yang, "A New Metaheuristic Bat-Inspired Algorithm," in Nature Inspired Cooperative Strategies for Optimization (NICSO 2010), vol. 284, J. R. Gonzalez, D. A. Pelta, C. Cruz, G. Terrazas and N. Krasnogor, Eds., Berlin, Springer, pp. 65-74, 2010, doi: 10.1007/978-3-642-12538-6_6.

[10] D. P. Rini S. M. Shamsuddin, and S. S. Yuhaniz, "Particle swarm optimization: technique, system and challanges," International Journal of Computer Applications, vol. 14, no. 1, pp. 19-27, January 2011, doi: 10.5120/1810-2331.

[11] J. Brest, A. Zamuda, I. Fister and M. S. Maučec, "Large scale global optimization using self-adaptive differential evolution algorithm," IEEE Congress on Evolutionary Computation, 2010, pp. 1-8, doi: 10.1109/CEC.2010.5585927.

[12] M. Kefayat, A. L. Ara, and S. A. N. Niaki, "A hybrid of ant colony optimization and artificial bee colony algorithm for probabilistic optimial placement and sizing of distributed energy resources," Energy Conversion and Management, vol. 92, pp. 149-161, March 2015, doi: 10.1016/j.enconman.2014.12.037.

[13] S. Kirkpatric, C. D. Gelatt, and M. P. Vecchi, “Optimization by Simulated Annealing,” Science, vol. 220, no. 4598, pp. 670- 680, May 1983, doi: 10.1126/science.220.4598.671.

[14] X. S. Yang, "Nature-inspired optimization algorithms: Challenges and open problems," Journal of Computational Science, vol. 46, no. 101104 , pp. 1-8, 2020, doi: 10.1016/j.jocs.2020.101104.

[15] M. I. Solihin and M. F. Zanil, "Performance comparison of cuckoo search and differential evolution algorithm for constrained optimization," Int. Eng. Research and Innov. Sym., vol. 160, no. 1, pp. 1-7, Nov. 2016, doi: 10.1088/1757-899X/160/1/012108

[16] W. Buaklee and K. Hongesombut, "Optimal DG allocation in a smart distribution grid using Cuckoo Search algorithm," 2013 10th Int. Conf. on Elect. Eng./Elect., Comp., Telecom. and Inf. Technology, 2013, pp. 1-6, doi: 10.1109/ECTICon.2013.6559624.

[17] S. K. Panda, P. Ray, and D. P. Mishra, "An efficient short-term electric power load forecasting using hybrid techniques," International Journal of Computer Information Systems and Industrial Management Applications, vol. 12, pp. 387- $397,2020$.

[18] S. K. Panda and P. Ray, "Analysis and evaluation of two short-term load forecasting techniques," International Journal of Emerging Electric Power Systems, 2021, pp. pp. 20210051, doi: 10.1515/ijeeps-2021-0051.

[19] S. S. Reddy, C. M. Jung and K. J. Seog, "Day-ahead electricity price forecasting using back propagation neural networks and weighted least square technique," Frontiers in Energy, vol. 10, no. 1, pp. 105-113, 2016, doi: 10.1007/s11708-016-0393-y.

[20] P. Ray, S. R. Arya, and S. Nandkeolyar, "Electric load forecasted by metaheuristic based back propagation approach," Journal of Green Engineering, vol. 7, pp. 61-82, 2017, doi: 10.13052/jge1904-4720.7124.

[21] M. Talaat, M. A. Farahat, N. Mansour, and A. Y. Hatata, "Load forecasting based on grasshopper optimization and a multilayer feed-forward neural network using regressive approach," Energy, vol. 196, pp. 1-12, 2020, doi: 10.1016/j.energy.2020.117087.

[22] M. Mansoor, F. Grimaccia, S. Leva, and M. Mussetta, "Comparison of echo state network and feed-forward neural networks in electrical load forecasting for demand response programs," Mathematics and Computers in Simulation, vol. 184, pp. $282-293,2021$.

[23] S. R. Salkuti, "Short-term electrical load forecasting using radial basis function neural networks considering weather factors," Electrical Engineering, vol. 100, no. 3, pp. 1985-1995, Sept. 2018, doi: 10.1007/s00202-018-0678-8.

[24] U. B. Tayaba, A. Zia, F. Yang, J. Lua, and M. Kashif, "Short-term load forecasting for microgrid energy management system using hybrid HHO-FNN model with best-basis stationary wavelet packet transform," Energy, vol. 203, pp. 1-11, 2020.

[25] A. Masoumi, F. Jabari, S. G. Zadeh, and B. Mohammadi-Ivatloo, "Long-term load forecasting approach using dynamic feedforward back-propagation artificial neural network," Optimization of Power System Problems, vol. 262, pp. 233-257, 2020. 
[26] G. F. Fan, Y. H. Guo, J. M. Zheng, and W. C. Hong, "A generalized regression model based on hybrid empirical mode decomposition and support vector regression with back-propagation neural network for mid-short-term load forecasting," Journal of Forecasting, vol. 9, no. 5, pp. 737-756, 2020, doi: 10.1002/for.2655.

[27] M. Kannaiyan, G. Karthikeyan, and J. G. T. Raghuvara, "Prediction of specific wear rate for LM25/ZrO2 composites using Levenberg-Marquardt backpropagation algorithm," Journal of Materials Research and Technology, vol. 9, no. 1, pp. 530-538, 2020, doi: 10.1016/j.jmrt.2019.10.082.

[28] S. S. Reddy, "Bat algorithm-based back propagation approach for short-term load forecasting considering weather factors," Electrical Engineering, vol. 100, no. 3, pp. 1297-1303, Sept. 2018, doi: 10.1007/s00202-017-0587-2

[29] W. Sun and C. Huang, "A carbon price prediction model based on secondary decomposition algorithm and optimized back propagation neural network," Journal of Cleaner Production, vol. 243, pp. 1-13, 2020, doi: 10.1016/j.jclepro.2019.118671.

[30] L. Wang, P. Wang, S. Liang, Y. Zhu, J. Khan, and S. Fang., "Monitoring maize growth on the North China Plain using a hybrid genetic algorithm-based back-propagation neural network model," Computers and Electronics in Agriculture, vol. 170, pp. 1-12, 2020, doi: 10.1016/j.compag.2020.105238.

[31] J. Su, Y. Chen, D. Zhang and Y. Kang, "Full-Parameter Identification Model Based on Back Propagation Algorithm for Brushless Doubly Fed Induction Generator," IEEE Transactions on Power Electronics, vol. 35, no. 10, pp. 9953-9958, Oct. 2020, doi: 10.1109/TPEL.2020.2976863.

[32] S. S. Reddy and C.M. Jung, "Short-term load forecasting using artificial neural networks and wavelet transform," International Journal of Applied Engineering Research, vol. 11, no. 19, pp. 9831-9836, Oct. 2016.

[33] Z. Wu, X. Zhao, Y. Ma, and X. Zhao, "Ahybrid modl based on modified multi-objective cuckoo search algorithm for short-term load forecasting," Applied Energy, vol. 237, pp. 896-909, 2019, doi: 10.1016/j.apenergy.2019.01.046.

[34] K. Xie, H. Yi, G. Hu, L. Li, and Z. Fan, "Short term power load forecasting based on Elman neural network with particle swarm optimization," Neurocomputing, vol. 416, pp. 136-142, 2020, doi: 10.1016/j.neucom.2019.02.063.

\section{BIOGRAPHIES OF AUTHORS}

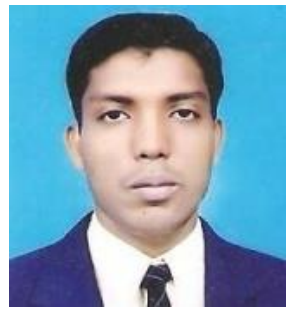

Saroj Kumar Panda (D) 8 sc $\mathrm{P}$ received the B.Tech in electrical and electronics engineering from Biju Patnaik University of Technology, Odisha, India in 2010 and the M.Tech in power electronics from Biju Patnaik University of Technology, Odisha, India in 2013 and pursuing the Ph.D. in power systems from Veer Surendra Sai University of Technology, Odisha, India. His research interests include the Application of Soft Computing Techniques in Power System Protection. He can be contacted at email: saroj.panda89@gmail.com.

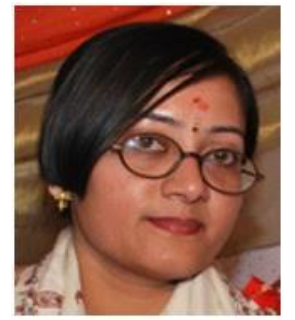

Papia Ray (iD) 8I SC P received the B.Tech in electrical engineering from Govt. College (Bihar University), India, M.Tech in power systems from Ranchi University, India and a Ph.D. degree in power systems from IIT, Delhi, India, in 2013. She is currently serving as Associate Professor in the Dept of Electrical Engg, Veer Surendra Sai University of Technology, Burla, Odisha. She has more than 17 years of teaching experience. She is the author of one book published by springer and more than 50 research articles. Further, she is the recipient of the Young Scientist award by DST, New Delhi in 2015. Her research interests include the Application of Soft Computing Techniques in Power System Protection, Wide-area measurement system, Biomedical Signal processing. She can be contacted at email: papiaray_ee@vssut.ac.in.

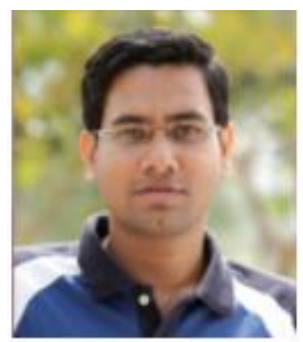

Debani Prasad Mishra (D) SC SC received the B.Tech. in electrical engineering from the Biju Patnaik University of Technology, Odisha, India, in 2006 and the M.Tech in power systems from IIT, Delhi, India in 2010. He has been awarded the Ph.D. degree in power systems from Veer Surendra Sai University of Technology, Odisha, India, in 2019. He is currently serving as Assistant Professor in the Dept of Elect. Eng, International Institute of Information Technology Bhubaneswar, Odisha. He has 11 years of teaching experience and 2 years of industry experience in the thermal power plant. He is the author of more than 80 research articles. His research interests include soft Computing techniques application in power system, signal processing and power quality. 3 students have been awarded Ph.D under his guidance and currently $4 \mathrm{Ph} . \mathrm{D}$. Scholars are continuing under him. He can be contacted at email: debani@iiit-bh.ac.in.

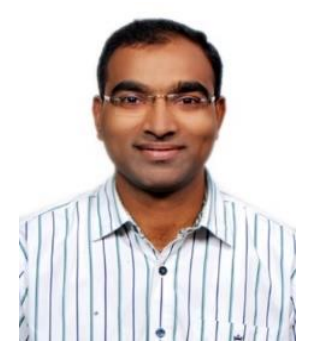

Surender Reddy Salkuti (D) 8 SC P received the Ph.D. degree in electrical engineering from the Indian Institute of Technology, New Delhi, India, in 2013. He was a Postdoctoral Researcher with Howard University, Washington, DC, USA, from 2013 to 2014. He is currently an Associate Professor with the Department of Railroad and Electrical Engineering, Woosong University, Daejeon, South Korea. His current research interests include power system restructuring issues, ancillary service pricing, real and reactive power pricing, congestion management, and market clearing, including renewable energy sources, demand response, smart grid development with integration of wind and solar photovoltaic energy sources, artificial intelligence applications in power systems, and power system analysis and optimization. He can be contacted at email: surender@wsu.ac.kr. 